\title{
Direct observation of optical trapping of a single quantum dot with an all-silicon nanoantenna
}

\author{
Zhe Xu1 ${ }^{1}$, Wuzhou Song ${ }^{1,2}$, and Kenneth B. Crozier ${ }^{1,3^{*}}$ \\ ${ }^{1}$ School of Physics, University of Melbourne, Victoria 3010, Australia \\ ${ }^{2}$ School of Materials Science and Engineering, Huazhong University of Science and Technology, Wuhan 430074, P. R. China \\ ${ }^{3}$ Department of Electrical and Electronic Engineering, University of Melbourne, Victoria 3010, Australia \\ Corresponding Author e-mail address: kenneth.crozier@unimelb.edu.au
}

\begin{abstract}
Silicon nanoantennas are used to optically trap individual streptavidin-coated CdSe/ZnS quantum dots (QDs) with minimal thermal effects. Using fluorescence microscopy, we track the position and light emission from optically-trapped QDs as a function of time.

OCIS codes: (140.7010) Laser trapping; (230.5590) Quantum-well, -wire and -dot devices;
\end{abstract}

\section{Introduction}

We demonstrate the optical trapping of single quantum dots (Life Technologies, Qdot 655 Streptavidin Conjugate, Q10121MP) with all-silicon (Si) nanoantennas, using illumination from a continuous wave (CW) trapping laser $(\lambda=1064 \mathrm{~nm})$. Interest in the optical trapping of QDs [1-4] has been motivated by the promise that QDs offer for applications in biomolecule labeling. Unlike previous reports, our all-Si nanoantennas present the opportunity for enhanced optical forces without deleterious thermal effects and complicated experimental set-ups. We furthermore directly observe the optical trapping of single quantum dots in vicinity of a nanoantenna, i.e. tracking their position vs time, for the first time to our knowledge. The $\mathrm{CdSe} / \mathrm{ZnS}$ core-shell QD is coated with a polymer and streptavidin, and has an overall diameter of $\sim 15-20 \mathrm{~nm}$ (Fig.1c). We present simulations of the optical forces, field distributions, and temperature rise occurring in optical trapping. We present experiments in which fluorescence microscopy is used to directly observe the trapping of individual QDs, enabling simultaneous monitoring of position and emission intensity vs time. We discuss the contributions of one- and two-photon absorption (TPA) to the emission.
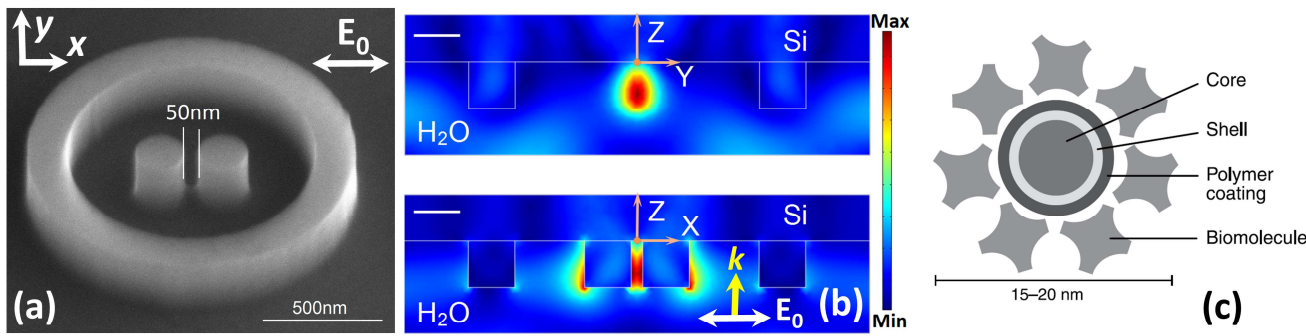

polymer coating
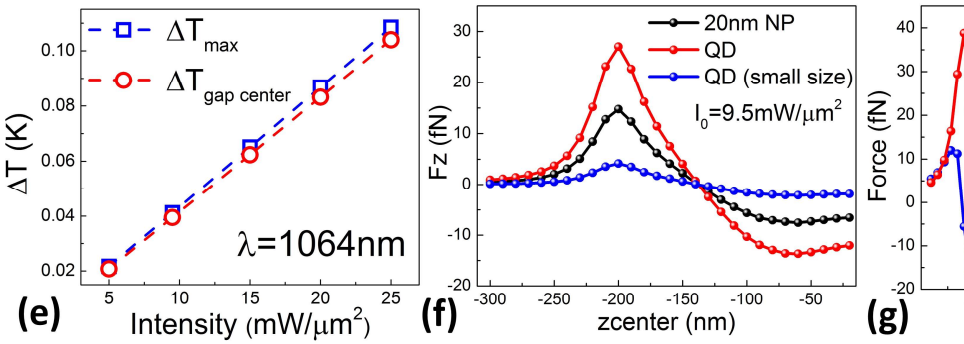

(c)

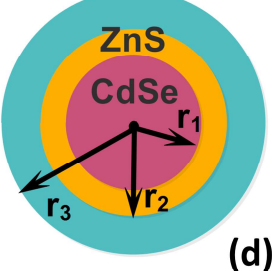

(d)

Fig.1. (a) SEM $45^{\circ}$-view image of Si nanoantenna. Each nanoantenna consists of two identical Si cylinders with diameters of $200 \mathrm{~nm}$, heights of $200 \mathrm{~nm}$ and a $50 \mathrm{~nm}$ gap in between, surrounded by an Si ring, all on an Si wafer substrate. Ring has inner and outer radii of 0.525 and $0.725 \mu \mathrm{m}$, respectively, and height $200 \mathrm{~nm}$. (b) Simulation of electric field $(|\mathrm{E}|)$ distribution in the $y z$ - and $x z$ - plane, for 1064 nm plane wave illumination (traveling in $+\mathrm{z}$ direction). Orange dots indicate coordinate system origin. Scale bar: $200 \mathrm{~nm}$. (c) Schematic illustration of QD structure [5]. (d) Model of QD used in optical force simulations. (e) Peak and gap center temperature increases $(\Delta T)$ vs trapping laser intensity. (f)\&(g) Optical forces as a function of position (f: $x_{\text {center }}=y_{\text {center }}=0$; g: $y_{\text {center }}=0, z_{\text {center }}=-215 \mathrm{~nm}$ ). NP: $d=20 \mathrm{~nm}, n=1.6$; QD: $r_{1}=5 \mathrm{~nm}, r_{2}=7 \mathrm{~nm}, r_{3}=10 \mathrm{~nm}$; Small QD: $r_{l}=3 \mathrm{~nm}, r_{2}=4 \mathrm{~nm}, r_{3}=5 \mathrm{~nm}$. QD trapping forces are determined on polymer surface. (h) Zoom-in of $|\mathrm{E}|$ distribution with a QD trapped in the gap.

\section{Numerical simulations}

Our nanoantenna comprises a pair of Si cylinders surrounded by an Si ring, all on an Si substrate (Fig.1a). The gap width $(50 \mathrm{~nm})$ is much larger than the QD diameter. We use a commercial finite element package (COMSOL) for the simulations. Illumination is from a plane wave $(x$-polarized, $\lambda=1064 \mathrm{~nm})$ incident from the water side. In Fig. $1 \mathrm{~b}$, we show the electric fields in two orthogonal cross sections. Three field maxima and trapping regions occur: a main 
maximum in the gap region, and two secondary maxima at the outer extremities of cylinders. In Fig. 1e, we plot the simulated temperature increase as a function of trapping laser intensity. It can be seen that both the peak temperature rise and the temperature rise in the gap center are both very small. In Fig.1f, we plot the vertical (i.e. into substrate) force $F_{z}$ on the QD as a function of its position in the vertical direction, found using the Maxwell stress tensor (MST) method. The particle is centered over the gap $\left(x_{\text {center }}=y_{\text {center }}=0\right)$. For comparison, calculations are included of the forces on a polystyrene nanoparticle (NP) and on a smaller QD. It can be seen that for $z_{\text {center }}<-140 \mathrm{~nm}$, each particle is attracted toward the antenna. The strongest forces are near the antenna edge $\left(z_{\text {center }}=-200 \mathrm{~nm}\right)$. In Fig. $1 \mathrm{~g}$, we plot $F_{x}$ and $F_{z}$ as functions of QD position along the $x$-axis $\left(y_{\text {center }}=0, z_{\text {center }}=-215 \mathrm{~nm}\right)$. In Fig. $1 \mathrm{~h}$, we show the electric field distribution ( $x z$-plane, $y=0$ ) when a QD is located at the vertical position $(z)$ for which $F_{z}=0$ (Fig.1f).

\section{Experimental results: optical trapping and two-photon excitation of individual QDs}

The QDs are suspended in phosphate-buffered saline solution (1X PBS, pH 7.4) with surfactant added (Tween 20, $0.05 \%(\mathrm{v} / \mathrm{v}))$ to prevent aggregation. The QD concentration is $\sim 10^{-8} \mathrm{~mol} / \mathrm{L}$. Our experimental set-up comprises an inverted optical microscope fitted with an oil immersion objective (NA 1.3), green (532 nm, for fluorescence excitation) and infrared (1064 nm, for trapping) lasers, filters, an electron-multiplying CCD (EM-CCD) camera and a spectrometer. In Fig.2a-c and Fig.2d-f, we present the results of two QD trapping experiments. We confirm that the QDs are not being stuck to the Si surface solely by some non-optical means by turning off the trapping laser and observing the QDs being released. In Fig.2a and 2d, we plot the fluorescence intensity counts integrated over a $30 \times 30$ pixel cross section centered over the Si nanoantenna of each EM-CCD frame as a function of time. A clear blinking behavior is observed during the period over which the QD is trapped ( 4-47s and $\sim 49-51 \mathrm{~s}$ in Fig.2a and $\sim 0$ $68 \mathrm{~s}$ in Fig.2d). Fig.2b shows an intensity count histogram of blinking trace (4-45s) in Fig.2a. A selection of four EM-CCD images of a trapped QD is shown as Fig.2c. Emission spectra of a single QD before and after switching off the green laser are also measured (Fig.2e). In Fig.2f, we present scatter plots of the positions of a trapped QD, for data sets obtained with both lasers and with trapping laser only. These are collected at a frame rate of $30 \mathrm{fps}$ and with $\sim 1000$ points per data set. These are from the same experiment as Fig.2d. In the experiments, due to the high TPA cross section $\left(\sigma_{T P A}\right)$, QDs can be excited by one-photon absorption from the green laser and/or TPA from the infrared laser. We confirm this via our EM-CCD images and from measured spectra (Fig.2e). We note however that at the (relatively weak) illumination level employed $\left(\sim 16.1 \mathrm{~mW} / \mu \mathrm{m}^{2}\right)$ and considering the intensity enhancement expected of our antenna $\left(\sim 4.91^{2}\right)$, the nonlinear refractive index $(\gamma \mathrm{I})$ of CdSe is $\sim-5.7 \times 10^{-7}\left(<<n_{0}=2.54\right)$, suggesting insignificant modification to the optical trapping force (Fig.1f\&g).

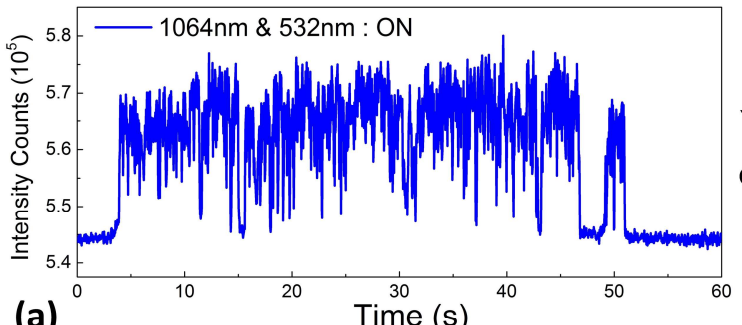

(a)

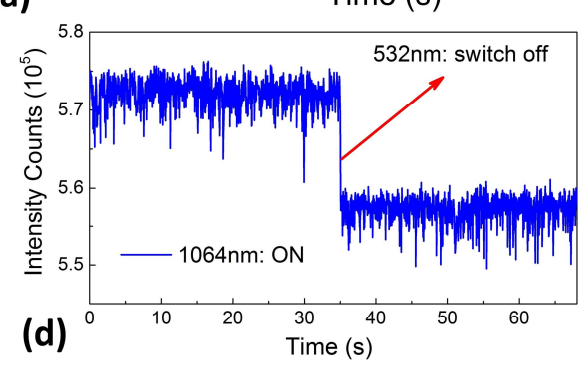

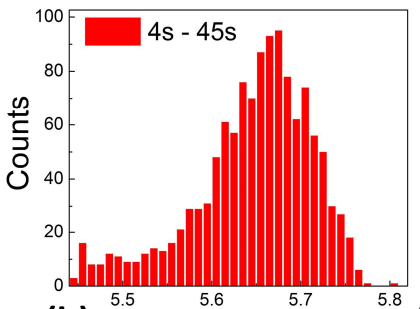

(b)
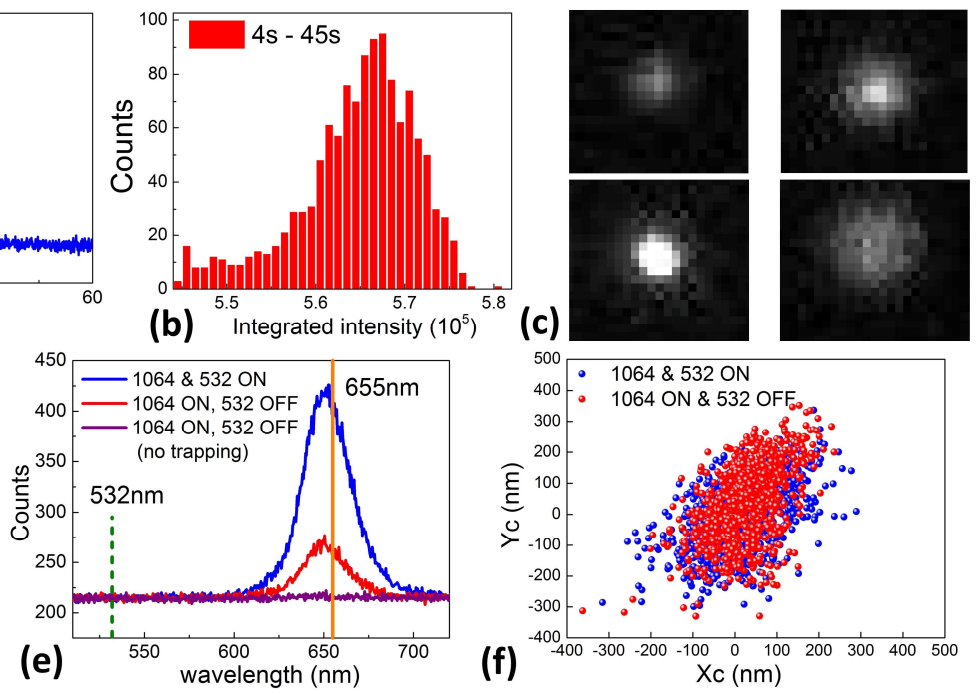

Fig.2. (a) Fluorescence intensity vs time for a single QD trapping, exposure time $\sim 30 \mathrm{~ms}$. (b) Corresponding histogram of fluorescence counts of QD in trapped state, extracted from data of panel a). (c) Selected EM-CCD frames showing fluorescent blinking of trapped QD. For panels a-c, $\mathrm{I}=17.3 \mathrm{~mW} / \mu^{2}$. (d) Experimental fluorescence time trace indicating optical trapping and two-photon excitation of a single QD. (e) Measured QD emission spectra (Integration time=3s). (f) Scatter plots of QD position, corresponding to two states in panel d). For panels $d-f$, $I=16.1 \mathrm{~mW} / \mu \mathrm{m}^{2}$.

\section{References}

[1] L. Jauffred, A.C. Richardson, and L.B. Oddershede. Nano Letters 8, 3376 (2008)

[2] Y. Tsuboi, et al. The Journal of Physical Chemistry Letters 1, 2327 (2010)

[3] Y.F. Chen, X. Serey, R. Sarkar, P. Chen, and D. Erickson. Nano Letters 12, 1633 (2012)

[4] R.A. Jensen, et al. ACS Photonics 3, 423 (2016)

[5] Invitrogen, https://tools.thermofisher.com/content/sfs/manuals/mp19000.pdf 